\title{
Tarifas e Taxas de Ocupação de Hoteis, Conforme suas Formas de Organização e Viajantes que Acolhem
}

\author{
Rates and Occupancy Rates of Hotels, According to their Forms of Organization and \\ Segment of Travelers They Host
}

\section{Tarifas y Tasas de Ocupación de Hoteles, de Acuerdo con su Organización y los Viajeros que Hospedan}

\author{
Eder Paschoal Pinto ${ }^{1}$ \\ Dárlei Geovanne Vianna dos Santos ${ }^{2}$
}

\begin{abstract}
Resumo
A exiguidade de estudos sobre apreçamento e ocupação em hoteis, estimulou os autores deste trabalho a verificar o relacionamento das ocupações obtidas pelos hoteis com suas tarifas, conforme suas formas de organização e os viajantes que acolhem, visando identificar relacionamentos estatisticamente significativos dentre e entre essas variáveis. A pesquisa foi realizada com os hoteis estabelecidos na localidade de Belo Horizonte, cuja cidade foi identificada como o quarto destino brasileiro mais procurado por executivos em 2010 . Gestores dos hoteis afiliados à Associação Brasileira de Indústria de Hoteis de Minas Gerais foram convidados para preencher o formulário de pesquisa. Quarenta e sete hoteis $(55 \%$ da população-alvo) gentilmente informaram os dados solicitados. Médias, desvios-padrão e coeficientes de correlação foram apurados. Os resultados indicaram que as tarifas diferem dentre e entre os segmentos de clientes que os hoteis acolhem, dentre os hoteis organizados em cadeia e entre eles e os hoteis independentes, e se relacionaram com as taxas de ocupação apenas em segmentos e meses específicos. Os detalhes apresentados neste artigo são úteis para gestores de hoteis, visto que eles podem compreender certas nuanças de apreçamento que aparentemente nada têm a ver com taxas de ocupação.
\end{abstract}

Palavras-chave: hotel; taxa de ocupação; tarifa; segmento de cliente; cadeia de hotel.

\footnotetext{
${ }^{1}$ Graduado, mestre, doutor e pós-doutor em Administração de Empresas: Mackenzie SP, PUC SP, FGV SP e Australian National University, respectivamente. Atuou no Mestrado em Administração da Universidade Salvador (grupo Laureate International Universities). Diretor-Consultor na Intelecto Consultoria S/C Ltda. Email: ederpinto@uol.com.br.

${ }^{2}$ Graduação em Administração de Empresas pelo Centro Universitário Newton Paiva, Especialização em Logística Integrada, Especialização em Administração Financeira e Mestrado em Administração. Gerente de Suprimentos do Serviço Nacional de Aprendizagem Comercial - Minas Gerais. E-mail: darlei@mg.senac.br.
} 


\begin{abstract}
The scarcity of studies on pricing and occupancy in hotels encouraged the authors of this article to verify the relationship between rates and occupancy rates, according to their forms of organization and segment of traveler they host, aiming to identify relationships statistically significant within and between these variables. The data were collected with the hotels located in the town of Belo Horizonte that has been identified as the fourth Brazilian destination most sought by executives in 2010. Managers of the hotel affiliated to the Brazilian Association of Hotel Industry of Minas Gerais were asked to fill out the form. Fortyseven hotels (55\% of the target population) kindly informed the required data. Means, standard deviations and correlations were calculated. The findings indicated that rates differ within and between segments of travelers that the hotel focus, as well as within of the hotel chains and between them and independent hotels; furthermore, rates correlate with occupancy rates only in specifics segments and months. The details presented in this article are useful for hotel managers, since they can understand certain nuances of pricing that seemingly have nothing to do with occupancy rates.
\end{abstract}

Keywords: hotel; occupancy rate; hotel rate; target marketing segment; hotel chain.

\title{
Resumen
}

La escasez de estudios sobre precios y ocupaciones en hoteles, estimuló a los autores de este trabajo para verificar el relacionamiento de las ocupaciones obtenidas por los hoteles con sus tarifas, de acuerdo consu organización y los viajeros que hospedan, para identificar relacionamientos estadisticamente significativas adentro y entre las variables. La pesquisa se llevó a cabo con los hoteles establecidos en la ciudad de Belo Horizonte, cuya ciudadfue identificado como el cuarto destino más popular en Brasil en 2010 por los ejecutivos. Los gerentes de los hoteles afiliados a la Asociación Brasileña de las Industrias de Hoteles de Minas Gerais se les pidieron responder alformulario de pesquisa. Cuarenta y siete hoteles (55\% de la población) informaron amablemente los datos solicitados. Medias, desviaciones estándary coeficientes de correlación fueran calculados. Los resultados indicaron que las tasas difieren entre y adentro de los segmentos de clientes, así como adentro de los hoteles dispuestos en cadenas y entre éstos y los hoteles independientes, y las tarifas se relacionan con las tasas de ocupación sólo en segmentos y meses especificos. Los datos aqui presentados sean útiles para los gerentes de los hoteles, ya que pueden comprender ciertos matices de los precios que aparentemente no tienen nada que ver con las tasas de ocupación.

Palabras clave: tasa de ocupación de los hoteles; tarifas, segmento de clientes; la cadena hotelera. 


\section{Introdução}

A literatura tem dado significativa importância à indústria hoteleira, principalmente por meio de pesquisas que analisaram os fatores que os hóspedes avaliam na escolha de um hotel (AGGETT, 2007; CARVELL; QUAN, 2008; CHAN; WONG, 2006; LOCKYER, 2005), no entanto poucos trabalhos têm relacionado esses fatores de escolha ao desempenho de hotéis (WILKINS; MERRILEES; HERINGTON, 2007) e, encontramos apenas um trabalho que no qual o autor analisou a relação entre tarifa e ocupação, a partir de dados extraídos de registros contábeis.

O estímulo essencial para a condução deste trabalho é, portanto, a necessidade de ampliar o escopo da pesquisa, conforme carência acima exposta. Adiciona-se, também o fato de que muitas cidades brasileiras irão sediar o campeonato mundial de futebol (denominado Copa do Mundo) de 2014, prevendo-se, portanto, a intensificação da entrada de turistas no Brasil e, como consequência, a necessidade de mais estudos com a indústria da hospitalidade.

Três núcleos de perguntas balizaram a pesquisa: Há diferenças nas tarifas médias aplicadas e nas ocupações obtidas pelos hoteis, dentre e entre os diversos segmentos de clientes que eles acolhem? Há diferenças nas tarifas médias aplicadas e nas ocupações obtidas dentre e entre os hoteis com distintas formas de organizar suas atividades (hoteis organizados em cadeias; hoteis independentes)? Em que extensão a tarifa aplicada pelo hotel determina sua taxa de ocupação?

No próximo tópico, haverá a aproximação das questões teóricas fundamentais à análise das descobertas e às considerações finais, em especial recorrendo-se a estudos empíricos realizados no setor hoteleiro. Na seção subsequente, o desenho da pesquisa será apresentado e, após, os dados obtidos serão apresentados e analisados. Nas considerações finais, as principais descobertas serão sintetizadas, sugestões para estudos serão elaboradas, e, por fim, será indicado o limite da aplicação das descobertas. 


\section{Aproximação do Apreçamento de Serviços Hoteleiros}

Lovelock e Wirtz (2010) definem sete componentes do mix de marketing de serviços, denominados 7 Ps (produto, praça, promoção, preço, pessoa, evidência física e processo). Chernev (2009) também descreve o mix com sete elementos: produto, serviço, preço, incentivos, comunicação, marca e distribuição. Boa parte da literatura, no entanto, descreve o composto de marketing com quatro elementos: ponto de venda, produto, preço e promoção (KOTLER, 2001). Assim, quer o composto de marketing seja formado por sete elementos, quatro, ou outra quantidade, o preço faz parte do composto.

O preço é um dos componentes mais sensíveis do composto mercadológico em virtude de influenciar diretamente a receita da firma (HOOLEY; SAUNDERS; PIERCY; NICOLAUD, 2008; PALIA, 2008) e, portanto, contribui com seu lucro (HOOLEY et. al., 2008; KOTLER, 2001), com sua imagem e com sua participação no mercado (NAGLE; HOGAN; ZALE, 2010). E, em virtude de sua complexidade requer atenção especial dos gestores (LAMB; HAIR JR; MCDANIEL, 2004), enquanto os outros componentes do composto de marketing geram custos (KOTLER, 2001; KOTLER; KELLER, 2008). O preço tende a ser elevado até o nível em que a diferenciação do produto a permite, no entanto, isso exige atenção ao impacto sobre o volume que pode ser vendido (KOTLER, 2001).

Para explicarem a importância do apreçamento, Rest e Harris (2008) comparam os papéis dos agentes mercadológicos com os do mercado: "Fixação de preços é uma competição entre o vendedor individual e o comprador individual. O mercado é o árbitro; os competidores a seção que zomba" (p. 178).

Para Nagle et al. (2010), marketing e apreçamento estão ligados por um cordão umbilical. Muitas organizações que ignoraram essa ligação aumentaram preços e, por mais paradoxal que pareça, diminuíram os seus lucros.

A tradicional concepção de preço orientada exclusivamente para garantir um adicional sobre os custos (lucro financeiro) pode ser ineficaz no médio e no longo prazo, uma vez que diante do preço está um mercado competidor dinâmico e um comprador que exige, cada vez mais, valor na relação comercial (CRESSMAN JR., 1999). 
Segundo Schwartz (2006), o viajante decide pelo hotel que apresenta a máxima utilidade, ou seja, se hospeda no hotel que apresenta mais e melhores benefícios em relação com a tarifa que tem de pagar. Para tanto, executa uma ou mais das seguintes estratégias: procura (pesquisa a melhor alternativa e, apenas depois de encontrá-la, efetua reserva); reserva e procura (faz a reserva e procura a melhor alternativa); reserva (e não faz nada mais); outro (decide por outro hotel).

Para se compreender e influenciar a decisão de compra do cliente, os pesquisadores Nagle et al. (2010) propõem a gestão de preços baseada em valor. De modo similar, Lehmann e Winer (2004) afirmam que a determinação do preço segundo o valor dado pelo cliente gera incremento de divisas, quando corretamente procedido. Nesse mesmo diapasão, Lovelock e Wright (2004) revelaram os resultados de uma pesquisa que lhes permitiram concluir que a estratégia-chave de apreçamento em serviços é associar "o preço que os clientes pagam ao valor que recebem" (p. 281).

Nasution e Mavondo (2008) conduziram uma pesquisa na Indonésia para verificar os valores que gestores hoteleiros acreditam oferecer aos seus clientes e os valores que os clientes experimentam. Os autores concluíram que, nas diferentes classes de hotéis, os valores que os clientes apreciam são distintos dos que os gestores acreditam deliberar.

A precificação de serviços hoteleiros tem como ponto de partida o segmento em que o hotel atua, isto é, os hoteis classificados como 'Luxo' aplicam tarifas superiores aos classificados como 'Turístico' e aos classificados como 'Econômico' e aos classificados como 'Simples'. (FERNANDES; BEDIA, 2004; PINTO, 2009). Em outras palavras, a tarifa aplicada depende da infraestrutura do estabelecimento, do tipo e da qualidade do serviço disponibilizado aos clientes, da localização do estabelecimento, bem como dos objetivos de custo e lucro (LOVELOCK; WIRTZ, 2010) e da possibilidade de utilizar a capacidade ociosa, principalmente na baixa temporada (BAUM; MUDAMBI, 1995).

$\mathrm{Na}$ época em que a coleta de dados foi realizada para esta pesquisa, a classificação, categorização e qualificação dos meios de hospedagem no Brasil eram simbolizadas por estrelas, de acordo com as condições de conforto, comodidade, serviços e atendimento que eles disponibilizam. Assim, o fator qualidade estava expresso, em termos oficiais, como: 
Super Luxo $(* * * * *$ SL), Luxo $(* * * * *)$; Superior $(* * * *)$; Turístico $(* * *)$; Econômico $(* *)$; Simples (*): Art. 11 do Regulamento do Sistema Oficial de Classificação de Meios de Hospedagem $^{3}$.

Em 09/12/2010 o Ministro do Turismo, Luiz Barreto, assinou portaria que Instituiu o Sistema Nacional de Classificação dos Meios de Hospedagem, mantendo as estrelas como símbolos, mas mudando as denominações para: Hotel, Pousada, Hotel-Fazenda, Hotel Histórico, Cama \& Café, Flat e Resort ${ }^{4}$.

Fernández e Bedia (2004) constataram que a quantidade de estrelas não é um fator infalível de determinação de sua qualidade. Eles compararam a classificação oficial dos hotéis de Cantabria, norte da Espanha, com as percepções que os clientes daqueles estabelecimentos expressaram sobre a qualidade de um hotel. Os autores concluíram que as classificações oficiais não correspondem às avaliações de qualidade processadas pelos clientes.

Em pesquisa com hoteis que atuam nos segmentos 'Turístico' e 'Luxo', estabelecidos no bairro Gonzaga, Santos, SP, Pinto (2009) verificou que as tarifas aplicadas pelos hoteis se correlacionam com suas taxas de ocupação. Isto é, houve uma relação negativa, tanto na amostra total, como nos segmentos de clientes: quanto maior a tarifa média aplicada, menor a taxa de ocupação média. Portanto, concluiu o autor, um aumento na tarifa praticada é acompanhado por uma redução na taxa de ocupação.

Por fim, ressalta-se que preço é um dos elementos que interage com os demais do composto de marketing na atração ou repulsa de clientes (LAMB et al., 2004). Por seu turno, para escolher o hotel para se hospedar, o viajante leva em consideração fatores, como: máxima utilidade - relação benefício-custo - (SCHWARTZ, 2006), sua experiência de compra (não necessariamente racional) e recomendações (CHAN; WONG, 2006). Assim, o gestor com reconhecido tino de liderança de mercado: 1) define a estrutura de grupos de clientes (segmentação) e delibera compostos mercadológicos para atender às particularizações

http://www.turismo.gov.br/export/sites/default/turismo/legislacao/downloads_legislacao/Regulamento_sistema_ oficial class meios hosp.pdf Acesso em 25/01/2011.

${ }^{4}$ http://www.turismo.gov.br/turismo/noticias/todas_noticias/20101208-4.html Acesso em 25/01/2011. 
(KOTLER; KELLER, 2008); 2) observa o momento da demanda, visto que os preços praticados no setor hoteleiro oscilam de acordo com os níveis e estrutura da demanda (BAUM; MUDAMBI, 1995); 3) empenha esforços para compreender a relação elasticidadepreço: compreensão fundamental para se tomar decisões de apreçamento (KOTLER, 2001); 4) define uma política duradoura de apreçamento e concede descontos apenas em situações especialíssimas, por exemplo, quando há capacidade ociosa, em virtude de descontos em preços não reterem clientes por muito tempo (CHAN; WONG, 2006) e; 5) analisa as atitudes e comportamentos dos concorrentes, bem como das outras forças de mercado, para definir o posicionamento estratégico competitivo, visto as guerrilhas existentes no setor, mormente em termos de preço. Para tanto, adota, entre outras, a estratégia de associar o preço que os clientes pagam ao valor que recebem (NAGLE et al., 2010). Entrementes, sabe que tal associação é altamente complexa e, para sua solução, são necessárias reiteradas interações com os clientes, principalmente para se conhecer e alinhar os diferentes valores/interesses (NASUTION; MAVONDO, 2008).

"Apostando" na aplicação dos princípios de marketing pelos gestores dos hoteis pesquisados, em especial, na gestão de preços/tarifas orientada para os distintos segmentos de clientes, os autores deste trabalho não adotaram hipóteses nulas, mas alternativas. Isto é, afirmaram que as tarifas médias e as taxas de ocupação apresentariam diferenças estatisticamente significativas nos diversos segmentos e nas duas formas de gestão. Afirmaram, também, que a tarifa se relacionaria negativamente com a ocupação, ou seja, quanto maior a tarifa média menor a taxa de ocupação.

\section{Arquitetura da Pesquisa}

O problema de pesquisa que gerou este estudo foi caracterizado como exploratório, visto que a literatura sobre o estudo do impacto da tarifa aplicada por hotéis - tendo como parâmetro os registros contábeis - em suas ocupações tem recebido pouca atenção dos acadêmicos: assim, é fundamental a continuidade desse tipo de estudo para que conclusões possam ser elaboradas. 
Em termos de localidade, a pesquisa é conclusiva, pois foi respondida por $55 \%$ da população hoteleira - Hair Jr., Wolfinbarger, Ortinau and Bush (2010) explicam que amostras aceitáveis do universo organizacional podem incluir apenas $30 \%$ da população. Destarte, pode-se dizer que a amostra participante da pesquisa é representativa da população-alvo.

É um estudo de campo, em virtude de levantar, tratar e analisar dados de registros contábeis de hotéis.

A natureza das variáveis é quantitativa e os resultados descrevem uma realidade ex-post facto. No Brasil, as pré-temporadas de turismo de lazer geralmente são em junho e dezembro e as temporadas são em julho e janeiro. Nas regiões em que o turismo mais intenso é o de eventos e negócios, a baixa temporada geralmente ocorre no fim de dezembro e no início de janeiro, sendo que os demais períodos são considerados de média ou alta temporada.

Decidiu-se cobrir um amplo cenário de temporadas, ou seja, as tarifas e as ocupações verificáveis nos registros contábeis dos hoteis de Belo Horizonte em um período de nove meses. A inspiração para investigar períodos de baixa, de média e de alta temporada emergiu do conhecimento dos resultados da pesquisa conduzida por Baum e Mudambi (1995). Suas descobertas revelaram que, em períodos de excesso de demanda, os preços são "bem comportados", no entanto, nos períodos de excesso de oferta, os preços não representam o estado da demanda.

A coleta de dados foi feita por meio de formulários enviados a todos os hotéis de Belo Horizonte inscritos na Associação Brasileira da Indústria de Hoteis de Minas Gerais. Após, seguiram correspondências eletrônicas, telefonemas e visitas, em quantidade moderada para não incomodar os participantes, entretanto, visando conseguir a maior adesão possível. Os contatos também visaram esclarecer dúvidas. O esforço propiciou resultado positivo, visto que a taxa de retorno foi de $55 \%$.

Em detalhes, foi solicitado ao respondente informar a classificação do hotel, o modelo de organização, as tarifas médias e as taxas de ocupação apuradas no período de dezembro/2008 a agosto/2009. 
A classificação informada é a que é determinada oficialmente, isto é, segundo o Art. 11 do Regulamento do Sistema Oficial de Classificação de Meios de Hospedagem. No entanto, foi reservado o direito de autoavaliação aos hoteis que não a possuíam.

O modelo de organização que foi requerido é o constante do contrato social do hotel, isto é, estar afiliado a uma cadeia (rede) ou organizado de forma isolada (independente).

A tarifa média foi apurada por meio da divisão do total da receita obtida no mês pela soma das unidades diariamente ocupadas.

A taxa de ocupação foi apurada da seguinte maneira: inicialmente apurou-se a taxa diária dividiu-se o total das unidades habitacionais ocupadas pelo total das unidades habitacionais existentes - e depois a mensal: foram somadas as taxas diárias e dividiu-se o resultado obtido pelo número de dias de cada mês.

Médias $^{5}$, desvios-padrão ${ }^{6}$ e coeficientes de correlação foram apurados, a partir dos dados obtidos. Em termos de correlação, primeiramente estudou-se o conjunto dos hotéis, sem segregá-los por segmento (amostra total); em seguida foi analisado cada um dos segmentos (Luxo, Superior, Turístico, Econômico e Simples) para verificar as distinções.

Os dados da pesquisa foram digitados e testados no software estatístico Sphinx Survey Edição Léxica versão 5.1.0.5. Esse software é produzido na França e representado no Brasil pela Sphinx Brasil e utilizado por diversas instituições educacionais e de outros setores. Isso é perceptível a partir dos clientes que a empresa divulga em seu site: Banco do Brasil, Ibope, Petrobrás, Sebrae, Unicamp, USP, dentre outros.

\section{Apresentação e Análise dos Dados Obtidos}

Nesta seção apresentam-se: 1) o perfil dos participantes da pesquisa; 2) os resultados dos testes realizados para apurar médias e desvios-padrão das tarifas e das ocupações nos

\footnotetext{
5 "Média: a média aritmética de todas as respostas; a soma de todos os valores de uma distribuição de repostas, dividida pelo número de respostas válidas" (HAIR Jr. et al., 2010, p.375).

6 "Desvio-padrão: Medida de dispersão média dos valores em um conjunto de respostas em relação à sua média" (HAIR Jr., et al., 2010, p.366).
} 
segmentos de clientes que os hoteis acolhem e segundo suas formas de organização e; 3) os resultados das regressões entre tarifa (variável independente) e ocupação (variável dependente).

\subsection{Perfil dos hoteis participantes da pesquisa}

Belo Horizonte é uma localidade que tem como principal impulsionador da ocupação hoteleira a realização de eventos e negócios. Essa capital foi o quarto destino brasileiro mais procurado por executivos em 2010, atrás de São Paulo, Rio de Janeiro e Porto Alegre, respectivamente, segundo a Carlson Wagonlit Travel (CWT), empresa especializada em gerenciamento de negócios ${ }^{7}$.

Várias cadeias de hoteis servem os viajantes que se destinam a Belo Horizonte, como: Accor, Arco Thess, Atlântica, Bristol, Clan, Comodoro, Nacional Inn, Othon, Royal Towers, Vetor Sul.

Foram convidados para participar da pesquisa 85 estabelecimentos hoteleiros cadastrados na Associação Brasileira da Indústria de Hotéis (ABIH) de Belo Horizonte, MG, Brasil.

Quarenta e sete hoteis $(55 \%$ da população) gentilmente preencheram o formulário de pesquisa, sendo suas classificações as seguintes: 11\% Luxo, 17\% Superior, 9\% Turístico, 44\% Econômico e, 19\% Simples. Em termos de formas de gestão, 58\% são organizados em cadeias e $42 \%$ não.

\subsection{Desempenho das tarifas e das taxas de ocupação nos segmentos}

Os resultados apresentados nesta seção e na seguinte (4.3) foram obtidos por meio do teste $t$, do teste $F$ e de regressão simples.

Hair Jr. et al. (2010) definem:

Teste $t$ : Procedimento de teste de hipóteses que utiliza a distribuição $t$ (p.382). Basicamente, o teste $t$ para diferenças entre médias de grupos pode ser conceitualizado como a diferença entre as medias dividida pela

\footnotetext{
http://www.carlsonwagonlit.com.br/pt/countries/br/noticias_e_imprensa/press_releases/release2010-08.html Acesso em 25/01/2011.
} 
variabilidade das médias. $\mathrm{O}$ teste $t$ apresenta um modo matemático de determinar se a diferença entre as duas médias amostrais ocorreu por acaso (p.283)

Teste $F$ : Teste usado para avaliar estatisticamente as diferenças entre médias de grupo em ANOVA (p. 382).

Os dados apresentados e analisados em seguida foram levantados a partir da seguinte questão: há diferenças nas tarifas aplicadas pelos hoteis, dentre e entre os diversos segmentos de clientes que eles acolhem? Assim, formulou-se a hipótese de que as tarifas diferem, dentre e entre os diversos segmentos.

A Tabela 1 e as notas que a seguem mostram que - exceto para os hoteis do segmento Econômico - houve em todos os meses, bem como na média aritmética das tarifas médias mensais, médias por categoria significativamente diferentes (teste $t$ ) do conjunto da amostra (ao risco de 95\%). Isto é, as tarifas médias diferem dentre e entre os segmentos de clientes que os hoteis acolhem, sendo que o segmento 'Luxo' apresenta a maior tarifa média (R\$192,00) e o segmento 'Simples' a menor $(\mathrm{R} \$ 51,00)$.

Tabela 1 - Desvios-Padrão e Médias das Tarifas Aplicadas Pelos Hoteis, Segundo suas Classificações

\begin{tabular}{|c|c|c|c|c|c|c|c|c|c|c|}
\hline to & 2008 & 2009 & 12009 & $\begin{array}{l}\text { Varço/ } \\
: 009\end{array}$ & $\begin{array}{l}\text { Abril/ } \\
2009\end{array}$ & $\begin{array}{l}\text { Jaio/ } \\
009\end{array}$ & $\begin{array}{l}\text { Junho/ } \\
2009\end{array}$ & $\begin{array}{l}\text { Julho/ } \\
2009\end{array}$ & $\begin{array}{l}\text { lgosto/ } \\
.009\end{array}$ & \\
\hline Lux & $\begin{array}{c}31,72 \\
(176,25)\end{array}$ & $\begin{array}{c}54,0 / \\
(170,50)\end{array}$ & $\begin{array}{c}19,62 \\
(183,75)\end{array}$ & $\begin{array}{c}25,49 \\
(199,50)\end{array}$ & $\begin{array}{c}26,04 \\
(191,75)\end{array}$ & $\begin{array}{c}20,68 \\
(190,75)\end{array}$ & $\begin{array}{c}27,66 \\
(195,25)\end{array}$ & $\begin{array}{c}31,65 \\
(203,50)\end{array}$ & $\begin{array}{c}35,33 \\
(216,00)\end{array}$ & $\begin{array}{c}27,36 \\
(192,00)\end{array}$ \\
\hline $\mathrm{S}$ & $\begin{array}{c}14,68 \\
(166,33)\end{array}$ & $\begin{array}{c}8,60 \\
(163,00)\end{array}$ & $\begin{array}{c}16,02 \\
(163,83)\end{array}$ & $\begin{array}{c}16,31 \\
(182,00)\end{array}$ & $\begin{array}{c}13,10 \\
(180,50)\end{array}$ & $\begin{array}{c}8,73 \\
(181,17)\end{array}$ & $\begin{array}{c}10,48 \\
(179,17\end{array}$ & $\begin{array}{c}9,16 \\
(177,50)\end{array}$ & $\begin{array}{c}12,75 \\
(187,83)\end{array}$ & $\begin{array}{c}10,52 \\
(175,67)\end{array}$ \\
\hline $\mathrm{Tu}$ & $\begin{array}{c}10,53 \\
(147,75)\end{array}$ & $\begin{array}{c}4,51 \\
(140,50)\end{array}$ & $\begin{array}{c}6,06 \\
(145,00)\end{array}$ & $\begin{array}{c}9,36 \\
(154,25)\end{array}$ & $\begin{array}{c}7,27 \\
(155,75)\end{array}$ & $\begin{array}{c}6,98 \\
(156,00)\end{array}$ & $\begin{array}{c}9,68 \\
(152,50)\end{array}$ & $\begin{array}{c}9,88 \\
(162,50)\end{array}$ & $\begin{array}{c}12,53 \\
(161,25)\end{array}$ & $\begin{array}{c}7,59 \\
(152,50)\end{array}$ \\
\hline $\begin{array}{l}\text { Econôm } \\
\text { ico }\end{array}$ & $(93,00)$ & $(92,06)$ & $(88,33)$ & $\begin{array}{c}24,01 \\
(97,39)\end{array}$ & $\begin{array}{c}22,44 \\
(101,61)\end{array}$ & $\begin{array}{c}24,49 \\
(104,50)\end{array}$ & $\begin{array}{c}21,44 \\
(101,50)\end{array}$ & $\begin{array}{c}21,88 \\
(103,56)\end{array}$ & $\begin{array}{c}22,47 \\
(103,61)\end{array}$ & $\begin{array}{c}20,71 \\
(98,44)\end{array}$ \\
\hline $\mathrm{S}$ & $\begin{array}{c}9,17 \\
(47,11)\end{array}$ & $\begin{array}{c}25,46 \\
(51,44)\end{array}$ & $\begin{array}{c}9,69 \\
(45,22)\end{array}$ & $\begin{array}{c}8,80 \\
(47,78)\end{array}$ & $\begin{array}{c}29,21 \\
(54,56)\end{array}$ & $\begin{array}{c}9,58 \\
(48,22)\end{array}$ & $\begin{array}{c}21,24 \\
(53,67)\end{array}$ & $\begin{array}{c}21,87 \\
(55,11)\end{array}$ & $\begin{array}{c}21,74 \\
(56,56)\end{array}$ & $\begin{array}{c}16,55 \\
(51,00)\end{array}$ \\
\hline & $\begin{array}{c}48,51 \\
(107,12)\end{array}$ & $\begin{array}{c}47,24 \\
(105,90)\end{array}$ & $\begin{array}{c}49,69 \\
(104,76)\end{array}$ & $\begin{array}{c}55,20 \\
(114,39)\end{array}$ & $\begin{array}{c}52,52 \\
(116,90)\end{array}$ & $\begin{array}{c}52,60 \\
(116,81)\end{array}$ & $\begin{array}{c}51,89 \\
(116,49)\end{array}$ & $\begin{array}{c}53,24 \\
(119,24)\end{array}$ & $\begin{array}{c}56,91 \\
(122,20)\end{array}$ & $\begin{array}{c}51,38 \\
(113,73)\end{array}$ \\
\hline
\end{tabular}

Notas: 
Média: Média aritmética das tarifas médias mensais; o montante da soma das tarifas médias mensais, dividido pelo número de meses (9).

Os valores da tabela são os desvios-padrão e as médias, calculadas sem considerar as não-respostas.

Os números grafados em negrito correspondem às médias por categorias significativamente diferentes (teste t) do conjunto da amostra (ao risco de $95 \%$ ).

Resultados do teste de Fisher:

Tarifa em dezembro/2008: entre $=20688.67$, dentre $=316.10, \quad \mathrm{~F}=65.45, \quad 1-\mathrm{p}=>99.99 \%$. Tarifa em janeiro/2009: entre $=17795.61$, dentre $=502.48, \quad \mathrm{~F}=35.42, \quad 1-\mathrm{p}=>99.99 \%$.

Tarifa em fevereiro/2009: entre $=22283.11$, dentre $=267.09, \quad \mathrm{~F}=83.43, \quad 1-\mathrm{p}=>99.99 \%$.

Tarifa em março/2009: entre $=26973.54$, dentre $=387.88, \quad \mathrm{~F}=69.54, \quad 1-\mathrm{p}=>99.99 \%$.

Tarifa em abril/2009: entre $=22976.53$, dentre $=512.21, \quad \mathrm{~F}=44.86, \quad 1-\mathrm{p}=>99.99 \%$.

Tarifa em maio/2009: entre $=24482.20$, dentre $=353.82, \quad \mathrm{~F}=69.19, \quad 1-\mathrm{p}=>99.99 \%$. Tarifa em junho/2009: entre $=23283.79$, dentre $=404.03, \quad \mathrm{~F}=57.63, \quad 1-\mathrm{p}=>99.99 \%$. Tarifa em julho/2009: entre $=24422.68$, dentre $=435.52, \quad \mathrm{~F}=56.08, \quad 1-\mathrm{p}=>99.99 \%$. Tarifa em agosto/2009: entre $=28035.59$, dentre $=483.23, \mathrm{~F}=58.02,1-\mathrm{p}=>99.99 \%$.

Média aritmética das tarifas mensais: entre $=23288.82$; dentre $=345.91, \mathrm{~F}=67.33,1-\mathrm{p}=>99.99 \%$.

A razão $\mathrm{F}$ expressa o resultado da divisão da variância entre os grupos sobre a variância dentro dos grupos e quanto maior a razão F maior a probabilidade de a hipótese nula ser rejeitada (HAIR Jr. et al., 2010).

Fonte: Resultados obtidos a partir dos dados informados pelos hoteis de Belo Horizonte.

Para verificar a correlação entre a média aritmética das tarifas médias mensais e segmentos de clientes que os hoteis acolhem, numeramos os hoteis conforme a quantidade de estrelas definidas em suas classificações. Isto é, os hoteis classificados como 'Simples' receberam uma estrela; os classificados como 'Econômico', duas; os segmentados como 'Turístico', três; os segmentados como 'Superior', quatro e; os classificados como 'Luxo', cinco.

O teste de regressão simples revelou: 1) que a dependência é muito significativa; 2) que quanto mais bem classificado é o hotel, maior a tarifa que aplica; 3) a equação da reta de regressão: tarifa média $=37,42$; pontuação categoria hotel $+21,55 ; 4)$ o coeficiente de correlação: $+0,92$ (pontuação categoria hotel explica 85\% da variância de tarifa média); 5) o desvio-padrão do coeficiente de regressão $(2,51)$.

Esses resultados confirmam a hipótese de que as tarifas se relacionam com os segmentos e atestam a validade da classificação oficial ou da autoavaliação feita por alguns hoteis, isto é, cada "estrela" inserida aumenta o valor da tarifa que clientes pagam. Esclareça-se, no entanto, que alguns hoteis reconsideraram a classificação inicialmente revelada no formulário de pesquisa, a partir de perguntas feitas pelos pesquisadores sobre a relação entre tarifa e classificação. Em detalhes, dentre os hoteis que informaram classificação no segmento 'Econômico', as tarifas aplicadas por cinco se enquadravam na faixa do segmento 'Simples'; 
dentre os hoteis que informaram classificação no segmento 'Turístico' quatro aplicavam tarifas que se enquadravam na faixa do segmento 'Econômico'.

Ressalta-se, no entanto, que o processo ocorreu de forma livre, visto que parte dos hoteis questionados manteve as informações (classificações) originais. Isto é, os pesquisadores não induziram os respondentes, mas apenas verificaram se as informações foram preenchidas corretamente - esse procedimento é salutar, pois, como exemplo, um hotel de uma rede internacional reconsiderou a classificação informada (Econômico) quando informamos que seu congênere informou tarifa idêntica e que estava classificado em grau distinto (Turístico).

Os dados apresentados e analisados em seguida foram levantados a partir da seguinte questão: há diferenças nas taxas de ocupação obtidas pelos hoteis, dentre e entre os diversos segmentos de clientes que eles acolhem? Assim, formulou-se a hipótese de que as ocupações diferem, dentre e entre os diversos segmentos.

A Tabela 2 e as notas que a seguem mostram que em nenhum dos meses, bem como na média aritmética das taxas mensais de ocupação, houve médias, por segmentos de clientes, significativamente diferentes (teste $t$ ) do conjunto da amostra (ao risco de 95\%). Em outras palavras, os hoteis dos diversos segmentos apresentam ocupações com diferenças estatisticamente não significativas.

Tabela 2 - Desvios-Padrão e Médias das Ocupações Obtidas Pelos Hoteis, Segundo suas Classificações

\begin{tabular}{lcccccccccc}
\hline Segment & Dez.l & Janeiro/ & Fevereiro/ & Março/ & Abril/ & Maio/ & Junho/ & Julho/ & Agosto/ & Média \\
0 & 2008 & 2009 & 2009 & 2009 & 2009 & 2009 & 2009 & 2009 & 2009 & \\
\hline \multirow{2}{*}{ Luxo } & 8,50 & 1,92 & 7,85 & 7,89 & 8,54 & 9,33 & 11,18 & 11,56 & 6,14 & 7,62 \\
& $(52,25)$ & $(48,50)$ & $(50,75)$ & $(63,75)$ & $(63,50)$ & $(66,50)$ & $(65,25)$ & $(70,50)$ & $(76,50)$ & $(62,00)$ \\
\multirow{2}{*}{ Superior } & 5,35 & 8,96 & 8,92 & 9,21 & 7,04 & 5,47 & 10,84 & 5,16 & 3,02 & 4,07 \\
& $(49,29)$ & $(43,57)$ & $(47,86)$ & $(62,86$ & $(59,43$ & $(63,43)$ & $(65,86)$ & $(70,29)$ & $(74,14)$ & $(59,71)$ \\
\multirow{2}{*}{ Turístico } & 8,58 & 14,17 & 6,48 & 6,48 & 6,76 & 6,98 & 8,96 & 8,66 & 5,25 & 6,95 \\
& $(56,25)$ & $(54,00)$ & $(52,00)$ & $(64,00)$ & $(64,50$ & $(70,00)$ & $(66,75)$ & $(74,50)$ & $(73,25)$ & $(63,75)$ \\
Econôm & 15,29 & 17,72 & 19,39 & 17,68 & 17,48 & 14,86 & 14,42 & 14,28 & 14,11 & 14,06 \\
ico & $(57,44)$ & $(54,28)$ & $(52,61)$ & $(63,50)$ & $(67,56$ & $(67,44)$ & $(66,06)$ & $(71,17)$ & $(73,28)$ & $(63,72)$ \\
Simples & 19,16 & 22,12 & 17,99 & 20,79 & 21,11 & 15,05 & 13,38 & 14,21 & 9,42 & 13,13 \\
\hline
\end{tabular}




\begin{tabular}{ccccccccccc}
\hline & $(46,22)$ & $(48,67)$ & $(41,11)$ & $(48,56)$ & $(62,33$ & $(61,22)$ & $(64,56)$ & $(65,56)$ & $(69,33)$ & $(56,11)$ \\
\hline \hline \multirow{2}{*}{ Total } & 14,34 & 16,40 & 16,10 & 16,50 & 15,47 & 12,59 & 12,40 & 12,25 & 10,51 & 11,63 \\
& $(53,07)$ & $(50,71)$ & $(49,12)$ & $(60,26)$ & $(64,41$ & $(65,60)$ & $(65,69)$ & $(70,07)$ & $(72,88)$ & $(61,26)$ \\
\hline
\end{tabular}

\section{Notas:}

Média: Média aritmética das tarifas médias mensais: o montante da soma das tarifas médias mensais, dividido pelo número de meses (9).

Os valores da tabela são os desvios-padrão e as médias, calculadas sem considerar as não-respostas.

Resultados do teste de Fisher:

Taxa de ocupação em dezembro/2008: entre $=227.46$, dentre $=203.32, \quad \mathrm{~F}=1.12, \quad 1-\mathrm{p}=63.72 \%$.

Taxa de ocupação em janeiro/2009: entre $=171.56$, dentre $=279.63, \quad \mathrm{~F}=0.61, \quad 1-\mathrm{p}=34.17 \%$.

Taxa de ocupação em fevereiro/2009: entre $=212.91$, dentre $=264.02, \mathrm{~F}=0.81,1-\mathrm{p}=46.89 \%$.

Taxa de ocupação em março/2009: entre $=393.45$, dentre $=259.25, \quad \mathrm{~F}=1.52, \quad 1-\mathrm{p}=78.38 \%$.

Taxa de ocupação em abril/2009: entre $=98.49$, dentre $=254.38, \quad \mathrm{~F}=0.39, \quad 1-\mathrm{p}=18.21 \%$.

Taxa de ocupação em maio/2009: entre $=86.85$, dentre $=166.24, \quad \mathrm{~F}=0.52, \quad 1-\mathrm{p}=27.76 \%$.

Taxa de ocupação em junho/2009: entre $=4.86$, dentre $=169.88, \quad \mathrm{~F}=0.03, \quad 1-\mathrm{p}=0.34 \%$.

Taxa de ocupação em julho/2009: entre $=71.16$, dentre $=158.60, \quad \mathrm{~F}=0.45, \quad 1-\mathrm{p}=22.52 \%$.

Taxa de ocupação em $/ 2009$ : entre $=45.05$, dentre $=117.41, \mathrm{~F}=0.38,1-\mathrm{p}=17.96 \%$.

Média aritmética das ocupações mensais: entre $=97.86$, dentre $=139.37, \mathrm{~F}=0.70,1-\mathrm{p}=40.20 \%$

Fonte: Resultados obtidos a partir dos dados informados pelos hoteis de Belo Horizonte.

Usando o mesmo artifício aplicado para verificar a relação entre a média aritmética das tarifas médias mensais e segmentos, apurou-se, por meio do teste de regressão simples, a relação entre a média aritmética das taxas mensais de ocupação e os segmentos dos hoteis, cujos resultados obtidos são: 1) a dependência não é significativa; 2) a equação da reta de regressão apresentou: ocupação média $=0,65$, pontuação categoria hotel $+59,63 ; 3)$ o coeficiente de correlação é $+0,07$ (pontuação categoria hotel explica $0 \%$ da variância de ocupação média); 4) o desvio-padrão do coeficiente de regressão é pouco influente $(1,44)$.

Os resultados confirmam, portanto, a aceitação da hipótese nula e rejeição da hipótese de que as ocupações diferem, dentre e entre os diversos segmentos.

\subsection{Tarifas e taxas de ocupação, conforme a forma do hotel se organizar}

Os dados apresentados e analisados em seguida foram levantados a partir da seguinte questão: há diferenças nas tarifas aplicadas, dentre e entre os hoteis com distintas formas de gerir suas atividades (hoteis organizados em cadeias; hoteis independentes)?

Formulou-se a hipótese de que as tarifas aplicadas pelos hoteis diferem dentre e entre os dois tipos de organização, em virtude de que as cadeias de hoteis tendem a estabelecer políticas nacionais de qualidade e precificação - conforme se pode verificar em seus sites. Os 
independentes, no entanto, podem precificar por métodos diversos - conforme os apontados na discussão teórica.

Tabela 3 - Desvios-padrão e Médias das Tarifas, Apurados a Partir dos Dados Obtidos com as Distintas Formas de Organização.

\begin{tabular}{|c|c|c|c|c|c|c|c|c|c|c|}
\hline Cadeia? & $\begin{array}{l}\text { Dez.l } \\
2008\end{array}$ & $\begin{array}{l}\text { Janeiro/ } \\
2009\end{array}$ & $\begin{array}{l}\text { Fevereiro/ } \\
2009\end{array}$ & $\begin{array}{l}\text { Março/ } \\
2009\end{array}$ & $\begin{array}{l}\text { Abril/ } \\
2009\end{array}$ & $\begin{array}{l}\text { Maio/ } \\
2009\end{array}$ & $\begin{array}{l}\text { Junho/ } \\
2009\end{array}$ & $\begin{array}{l}\text { Julho/ } \\
2009\end{array}$ & $\begin{array}{l}\text { Agosto/ } \\
2009\end{array}$ & Média \\
\hline Sim & $\begin{array}{c}38,42 \\
(142,47)\end{array}$ & $\begin{array}{c}38,76 \\
(139,73)\end{array}$ & $\begin{array}{c}40,84 \\
(142,67)\end{array}$ & $\begin{array}{c}48,05 \\
(157,00)\end{array}$ & $\begin{array}{c}43,59 \\
(155,73)\end{array}$ & $\begin{array}{c}40,22 \\
(157,13)\end{array}$ & $\begin{array}{c}42,96 \\
(153,87\end{array}$ & $\begin{array}{c}44,76 \\
(155,93\end{array}$ & $\begin{array}{c}48,16 \\
(162,73)\end{array}$ & $\begin{array}{c}42,24 \\
(151,87)\end{array}$ \\
\hline Não & $\begin{array}{c}42,72 \\
(86,28)\end{array}$ & $\begin{array}{c}41,41 \\
(86,36)\end{array}$ & $\begin{array}{c}41,51 \\
(82,60)\end{array}$ & $\begin{array}{c}44,03 \\
(89,48)\end{array}$ & $\begin{array}{c}44,15 \\
(93,08)\end{array}$ & $\begin{array}{c}45,41 \\
(93,24)\end{array}$ & $\begin{array}{c}45,03 \\
(94,48)\end{array}$ & $\begin{array}{c}47,14 \\
(97,60)\end{array}$ & $\begin{array}{c}49,11 \\
(98,12)\end{array}$ & $\begin{array}{c}43,82 \\
(91,24)\end{array}$ \\
\hline Total & $\begin{array}{c}49,11 \\
(107,35)\end{array}$ & $\begin{array}{c}47,75 \\
(106,38)\end{array}$ & $\begin{array}{c}50,26 \\
(105,13)\end{array}$ & $\begin{array}{c}55,84 \\
(114,80)\end{array}$ & $\begin{array}{c}53,15 \\
(116,58)\end{array}$ & $\begin{array}{c}53,21 \\
(117,20)\end{array}$ & $\begin{array}{c}52,52 \\
(116,75)\end{array}$ & $\begin{array}{c}53,90 \\
(119,48)\end{array}$ & $\begin{array}{c}57,62 \\
(122,35)\end{array}$ & $\begin{array}{c}52,01 \\
(113,98)\end{array}$ \\
\hline
\end{tabular}

Notas:

Média: Média aritmética das tarifas médias mensais: o montante da soma das tarifas médias mensais, dividido pelo número de meses (9).

Os valores da tabela são os desvios-padrão e as médias, calculadas sem considerar as não-respostas.

Os números grafados em negrito correspondem às médias por categorias significativamente diferentes (teste $t$ ) do conjunto da amostra (ao risco de $95 \%$ ).

Resultados do teste de Fisher:

Tarifas em dezembro/2008: entre $=29598.41, \quad$ dentre $=1652.64, \quad \mathrm{~F}=17.91, \quad 1-\mathrm{p}=99.98 \%$

Tarifas em janeiro/2009: entre $=26715.61$, dentre $=1594.84, \quad \mathrm{~F}=16.75, \quad 1-\mathrm{p}=99.97 \%$

Tarifas em fevereiro/2009: entre $=33830.49$, dentre $=1658.96, \quad \mathrm{~F}=20.39, \quad 1-\mathrm{p}=99.99 \%$.

Tarifas em março/2009: entre $=42746.88$, dentre $=2021.60, \quad \mathrm{~F}=21.15, \quad 1-\mathrm{p}=99.99 \%$.

Tarifas em abril/2009: entre $=36805.29$, dentre $=1881.25, \quad \mathrm{~F}=19.56, \quad 1-\mathrm{p}=99.99 \%$.

Tarifas em maio/2009: entre $=38278.35$, dentre $=1849.70, \quad \mathrm{~F}=20.69, \quad 1-\mathrm{p}=99.99 \%$.

Tarifas em junho/2009: entre $=33066.28$, dentre $=1910.36, \quad \mathrm{~F}=17.31, \quad 1-\mathrm{p}=99.98 \%$

Tarifas em julho/2009: entre $=31903.18, \quad$ dentre $=2086.69, \quad \mathrm{~F}=15.29, \quad 1-\mathrm{p}=99.96 \%$.

Tarifas em agosto/2009: entre $=39140.49$, dentre $=2316.91, \mathrm{~F}=16.89,1-\mathrm{p}=99.98 \%$.

Média aritmética das tarifas mensais: entre $=34461.05$, dentre $=1821.85, \mathrm{~F}=18.92,1-\mathrm{p}=99.99 \%$.

Fonte: Resultados obtidos a partir dos dados informados pelos hoteis de Belo Horizonte.

A Tabela 3 e as notas que a seguem mostram que os hoteis organizados em cadeias apresentam, em todos os meses e na média aritmética das tarifas médias mensais, médias por categorias significativamente diferentes do conjunto da amostra (ao risco de 95\%). Isto é, as tarifas são significativamente diferentes no conjunto desses hoteis e superiores às dos hoteis independentes. O conjunto dos hoteis independentes, por seu turno, apresenta em seu conjunto diferenças estatisticamente significativas nos meses de fevereiro/2009 a abril/2009.

Os resultados apontam para a rejeição da hipótese nula - ausência de diferença - e aceitação da hipótese alternativa, isto é, as tarifas aplicadas pelos hoteis organizados em cadeias apresentam diferenças estatisticamente significativas dentre eles, bem como diferem 
das aplicadas pelos hoteis independentes. Dentre esses (independentes), entretanto, não há indicação de assiduidade das diferenças.

Os dados apresentados e analisados em seguida foram levantados a partir da seguinte questão: há diferenças nas taxas de ocupação obtidas dentre e entre os hoteis com distintas formas de organização? Assim, formulou-se a hipótese de que as ocupações obtidas pelos hoteis diferem dentre e entre as duas formas de organizar suas atividades.

Tabela 4 - Desvios-padrão e Médias das Ocupações, Apurados a Partir dos Dados Obtidos com as Distintas Formas de Organização.

\begin{tabular}{|c|c|c|c|c|c|c|c|c|c|c|}
\hline \multirow[t]{2}{*}{ Cadeia? } & Dez.l & Janeiro/ & Fevereiro/ & Março/ & Abril/ & Maio/ & Junho/ & Julho/ & Agosto/ & \multirow[t]{2}{*}{ Média } \\
\hline & 2008 & 2009 & 2009 & 2009 & 2009 & 2009 & 2009 & 2009 & 2009 & \\
\hline \multirow[b]{2}{*}{ Sim } & 13,65 & 15,41 & 11,56 & 11,66 & 12,45 & 11,64 & 11,74 & 10,99 & 5,63 & 9,83 \\
\hline & $(56,94)$ & $(52,38)$ & $(53,06)$ & $(63,63)$ & $(63,44)$ & $(66,19)$ & $(66,31)$ & $(72,38)$ & $(75,50)$ & $(63,31)$ \\
\hline \multirow{2}{*}{ Não } & 14,79 & 17,51 & 18,01 & 18,74 & 17,48 & 13,55 & 13,24 & 13,17 & 12,66 & 12,76 \\
\hline & $(50,72)$ & $(49,44)$ & $(45,88)$ & $(57,40)$ & $(64,56)$ & $(64,96)$ & $(65,12)$ & $(68,40)$ & $(71,08)$ & $(59,64)$ \\
\hline \multirow{2}{*}{ Total } & $\overline{14,51}$ & 10,59 & 16,04 & 16,47 & $\overline{10,55}$ & 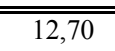 & $\overline{12,54}$ & $\overline{12,38}$ & $\overline{10,62}$ & $\overline{111,71}$ \\
\hline & $(53,15)$ & $(50,59)$ & $(48,68)$ & $(59,83)$ & $(64,12)$ & $(65,44)$ & $(65,59)$ & $(69,95)$ & $(72,81)$ & $(61,07)$ \\
\hline
\end{tabular}

Notas:

Média: Média aritmética das tarifas médias mensais: o montante da soma das tarifas médias mensais, dividido pelo número de meses (9). Os valores da tabela são os desvios-padrão e as médias, calculadas sem considerar as não-respostas.

Resultados do teste de Fisher:

Ocupação em dezembro/2008: entre $=377.38, \quad$ dentre $=201.15, \quad \mathrm{~F}=1.88, \quad 1-\mathrm{p}=82.49 \%$. Ocupação em janeiro/2009: entre $=84.72, \quad$ dentre $=273.00, \quad \mathrm{~F}=0.31, \quad 1-\mathrm{p}=41.28 \%$

Ocupação em fevereiro/2009: entre $=511.10$, dentre $=244.74, \quad \mathrm{~F}=2.09, \quad 1-\mathrm{p}=84.75 \%$. Ocupação em março/2009: entre $=385.73$, dentre $=261.64, \quad \mathrm{~F}=1.47, \quad 1-\mathrm{p}=77.01 \%$. Ocupação em abril/2009: entre $=15.57$, dentre $=241.40, \quad \mathrm{~F}=0.07, \quad 1-\mathrm{p}=21.12 \%$. Ocupação em maio/2009: entre $=15.70$, dentre $=161.04, \quad \mathrm{~F}=0.10, \quad 1-\mathrm{p}=24.91 \%$. Ocupação em junho/2009: entre $=14.33$, dentre $=156.80, \quad \mathrm{~F}=0.09, \quad 1-\mathrm{p}=24.26 \%$. Ocupação em julho/2009: entre $=154.75$, dentre $=149.34, \quad \mathrm{~F}=1.04, \quad 1-\mathrm{p}=68.39 \%$. Ocupação em agosto/2009: entre $=190.84$, dentre $=108.10, \mathrm{~F}=1.77,1-\mathrm{p}=81.16 \%$.

Média aritmética das ocupações mensais: entre $=133.04$, dentre $=133.88, \mathrm{~F}=0.99,1-\mathrm{p}=67.35 \%$.

Fonte: Resultados obtidos a partir dos dados informados pelos hoteis de Belo Horizonte.

A Tabela 4 e as notas que a seguem mostram que em nenhum mês, bem como na média aritmética das taxas mensais de ocupação, houve média por categoria significativamente diferente do conjunto da amostra (ao risco de 95\%).

Os resultados apontam para a aceitação da hipótese nula e rejeição da hipótese alternativa, isto é, as ocupações apresentam diferenças estatisticamente não significativas dentre e entre os hoteis organizados de formas distintas. 


\subsection{Relação entre tarifa média e taxa de ocupação}

Os resultados apresentados nesta seção foram apurados por meio do método de Pearson, isto é,

O coeficiente de correlação de Pearson mede o grau de associação linear entre duas variáveis. $O$ coeficiente varia de $-1,00$ a 1,00 , sendo que 0 representa a absoluta ausência de associação entre duas variáveis e $-1,00 \mathrm{ou}$ 1,00 representam uma relação perfeita entre ambas [...] A hipótese nula para o coeficiente de correlação de Pearson é que não há associação entre as duas variáveis e o coeficiente de correlação é igual a zero (HAIR JR.; et al., 2010, p. 310).

O objetivo do método de Pearson é verificar a força da significância e o tipo de relação. Hair Jr. et al. (2010) definem "as regras práticas sobre a força dos coeficientes de correlação", conforme a amplitude do coeficiente: 0.81 a 1.00 , muito forte; 0.61 a 0.80 , forte; 0.41 a 0.60 , moderado; 0.21 a 0.40, fraco; 0.00 a 0.20, fraco sem relação (p. 311). As relações entre variáveis podem ser positivas ou negativas. "Uma relação positiva entre duas variáveis é aquela em que as duas variáveis crescem ou decrescem juntas [...] As relações negativas sugerem que, enquanto uma variável aumenta, a outra diminui” ( $p$ 76).

Os dados apresentados e analisados em seguida foram levantados a partir da seguinte questão: em que extensão a tarifa aplicada pelo hotel determina sua taxa de ocupação?

Tabela 5 - Extensão em que as Tarifas Explicam as Taxas de Ocupação.

\begin{tabular}{|c|c|c|c|c|c|c|c|c|c|c|}
\hline Segment & $\begin{array}{l}\text { Dez./ } \\
2008\end{array}$ & $\begin{array}{l}\text { Janeiro/ } \\
2009\end{array}$ & $\begin{array}{l}\text { Fevereiro/ } \\
2009\end{array}$ & $\begin{array}{l}\text { Março/ } \\
2009\end{array}$ & $\begin{array}{l}\text { Abril/ } \\
2009\end{array}$ & $\begin{array}{l}\text { Maio/ } \\
2009\end{array}$ & $\begin{array}{l}\text { Junho/ } \\
2009\end{array}$ & $\begin{array}{l}\text { Julho/ } \\
2009\end{array}$ & $\begin{array}{l}\text { Agosto/ } \\
2009\end{array}$ & Média \\
\hline Amostra & $0 \%$ & $0 \%$ & $1 \%$ & $4 \%$ & $0 \%$ & $0 \%$ & $0 \%$ & $1 \%$ & $1 \%$ & $0 \%$ \\
\hline total & DNS & DNS & DNS & DNS & DNS & DNS & DNS & DNS & DNS & DNS \\
\hline \multirow{2}{*}{ Luxo } & $77 \%$ & $25 \%$ & $30 \%$ & $11 \%$ & $93 \%$ & $88 \%$ & $70 \%$ & $53 \%$ & $34 \%$ & $63 \%$ \\
\hline & DS- & DPS & DPS & DNS & DMS- & DMS- & DS- & DM- & DPS & DS- \\
\hline \multirow{2}{*}{ Superior } & $0 \%$ & $77 \%$ & $17 \%$ & $2 \%$ & $4 \%$ & $4 \%$ & $53 \%$ & $10 \%$ & $21 \%$ & $24 \%$ \\
\hline & DNS & DS+ & DNS & DNS & DNS & DNS & $\mathrm{DM}^{+}$ & DNS & DPS & DPS \\
\hline Turístic & $97 \%$ & $79 \%$ & $57 \%$ & $73 \%$ & $41 \%$ & $80 \%$ & $94 \%$ & $27 \%$ & $71 \%$ & $74 \%$ \\
\hline o & DMS- & DS- & DM- & DS- & DM- & DS- & DMS- & DPS & DS- & DS- \\
\hline Econômi & $0 \%$ & $0 \%$ & $2 \%$ & $0 \%$ & $1 \%$ & $3 \%$ & $1 \%$ & $2 \%$ & $0 \%$ & $0 \%$ \\
\hline co & DNS & DNS & DNS & DNS & DNS & DNS & DNS & DNS & DNS & DNS \\
\hline
\end{tabular}




\begin{tabular}{lllllllllll}
\hline \multirow{2}{*}{ Simples } & $0 \%$ & $0 \%$ & $6 \%$ & $13 \%$ & $1 \%$ & $20 \%$ & $12 \%$ & $7 \%$ & $17 \%$ & $8 \%$ \\
& DNS & DNS & DNS & DNS & DNS & DNS & DNS & DNS & DNS & DNS \\
\hline
\end{tabular}

Notas:

Média: Média aritmética das taxas mensais de ocupação: o montante da soma das taxas mensais de ocupação, dividido pelo número de meses (9).

Amplitude do coeficiente: 0.81 a 1.00 , muito forte/muito significativa; 0.61 a 0.80 , forte/significativa; 0.41 a 0.60 , moderado; 0.21 a 0.40 , fraco/pouco significativa; 0.00 a 0.20 , fraco sem relação/não significativa. Amplitude transformada em porcentagem; por exemplo, amplitude $0.71=$ a $71 \%$.

Siglas: DNS: dependência não significativa. DPS: dependência pouco significativa. DM: dependência moderada. DS: dependência significativa. DMS: dependência muito significativa.

(+) Relação positiva: Significa que quanto maior é a tarifa, maior a taxa de ocupação.

(-) Relação negativa: Significa que quanto maior a tarifa, menor a taxa de ocupação.

Conforme demonstrados na Tabela 5, os resultados obtidos nos testes realizados com a amostra total e com os hoteis classificados como 'Simples' e 'Econômico' revelaram dependências não significativas (fracas sem relações) entre tarifas médias e taxas de ocupação.

Em oito meses, dentre os nove pesquisados, as taxas de ocupação dos hoteis do segmento 'Turístico' resultaram de suas tarifas, isto é, maiores as tarifas, menores as taxas de ocupação (duas associações moderadas, quatro fortes e duas muito fortes). $\mathrm{O}$ teste realizado com as médias aritméticas confirmou a relação negativa: em termos precisos, as tarifas explicaram $74 \%$ da variação nas taxas de ocupação (amplitude forte). Entrementes, em termos das características do teste aplicado, é preciso cautela para aceitar esses resultados em virtude do pequeno número de hoteis pertencentes ao segmento.

Nos hotéis classificados como 'Superior', em dois meses as taxas de ocupação resultaram das tarifas (uma associação moderada e uma forte) e apresentaram relações positivas, ou seja, maiores as tarifas médias aplicadas, maiores as taxas de ocupação. Se isso fosse observado na totalidade ou na maioria dos meses, os gestores dos hoteis desse segmento poderiam aumentar suas tarifas com a esperança de aumento nas taxas de ocupação. No entanto, além de $78 \%$ dos meses mostrarem relações estatisticamente não, ou pouco significativas, o teste realizado com as médias aritméticas apresentou dependência pouco significativa: a tarifa explica $24 \%$ da variação da ocupação.

Em cinco meses, dentre os nove pesquisados, as tarifas aplicadas pelos hoteis do segmento 'Luxo' determinaram as suas taxas de ocupação (uma associação moderada, duas associações 
fortes e duas muito fortes), ou seja, maiores as tarifas aplicadas, menores as taxas de ocupação. O teste realizado com as médias aritméticas confirmou a relação negativa: em termos precisos, as tarifas explicaram 63\% da variação das taxas de ocupação (amplitude forte). Entrementes, em termos das características do teste aplicado, é preciso cautela para aceitar esses resultados em virtude do pequeno número de hoteis pertencentes ao segmento.

Os resultados obtidos nos segmentos 'Luxo' e 'Turístico' corroboram a descoberta de Pinto (2009) e apóiam as descobertas de pesquisas com viajantes que apontaram a tarifa dentre os principais elementos do composto de marketing a influenciar suas decisões de escolha de um hotel (CARVELL; QUAN, 2008; CHUNG, 2000; LOCKYER, 2005; SCHWARTZ; CHEN, 2010).

As relações negativas (quanto maior a tarifa, menor a ocupação) apontam para a sensibilidade dos clientes às tarifas, isto é, cada unidade de preço ampliada tende a gerar uma unidade inferior na taxa de ocupação. Semelhante descoberta foi apontada por Pinto (2009), isto é, ele verificou relações negativas nesses mesmos segmentos (Luxo e Turístico) em pesquisa com os hoteis do bairro do Gonzaga, Santos, SP, isto é, "quanto maior foi a tarifa média aplicada menor a ocupação média” (p. 479). Várias outras pesquisas também comprovaram sensibilidade ao preço. Por exemplo, estudando a lealdade à marca e sensibilidade ao preço entre grupos de consumidores na escolha da marca, Botelho e Urdan (2005) constataram que "a diferença de sensibilidade ao preço entre os consumidores varia de acordo com as marcas" (p. 163). Analogamente, há descobertas que revelam a identificação do cliente com a marca que ele escolhe, isto é, pesquisando o valor da marca, Kim e Kim (2005) verificaram um relacionamento positivo dos valores dos clientes com os desempenhos de hoteis de luxo.

\subsection{Tarifas médias e taxas de ocupação nos segmentos 'Luxo' e 'Turístico', conforme o tipo de organização}

A descoberta das relações negativas entre as taxas de ocupação e as tarifas médias aplicadas pelos hoteis dos segmentos 'Luxo' e 'Turístico' estimulou-nos a verificar se há diferenças significativas dentre essas variáveis, segundo o tipo de organização. 
Assim, a seguinte pergunta de pesquisa emergiu: os hoteis dos segmentos 'Luxo' e 'Turístico' que estão organizados em cadeias aplicam tarifas e obtêm taxas de ocupação distintas dos hoteis independentes?

Tabela 6 - Médias Aritméticas Verificadas nos Hoteis Organizados em Cadeias e nos Hoteis Independentes, do Segmento 'Luxo'

\begin{tabular}{lcc}
\hline Hotel Organizado em Cadeia? & $\begin{array}{c}\text { Média Aritmética das } \\
\text { Tarifas Médias Mensais }\end{array}$ & $\begin{array}{c}\text { Média Aritmética das Taxas Mensais de } \\
\text { Ocupação }\end{array}$ \\
\hline Sim & 190 & 65 \\
Não & 198 & 54 \\
\hline \hline Total & 192 & 62 \\
\hline
\end{tabular}

Notas:

Média aritmética das tarifas médias mensais: O montante da soma das tarifas médias mensais, dividido pelo número de meses (9).

Média aritmética das taxas mensais de ocupação: $\mathrm{O}$ montante da soma das taxas mensais de ocupação, dividido pelo número de meses (9).

Os valores da tabela são os desvios-padrão e as médias, calculadas sem considerar as não-respostas.

Resultados do teste de Fisher:

Média aritmética das tarifas mensais: entre $=48.00$, dentre $=2.198 .00, \mathrm{~F}=0.02,1-\mathrm{p}=9.64 \%$ Média aritmética das ocupações mensais: entre $=85.33$, dentre $=88.67, F=0.96,1-p=49.41 \%$ Fonte: Resultados obtidos a partir dos dados informados pelos hoteis de Belo Horizonte.

Os resultados expressos na Tabela 6 revelam diferenças estatisticamente não significativas das tarifas e das ocupações entre os dois tipos de organização (hoteis em cadeias; hoteis independentes) do segmento 'Luxo'.

Semelhantemente às descobertas verificadas com os hoteis do segmento 'Luxo', os resultados digitados na Tabela 7 apontam para diferenças estatisticamente não significativas das tarifas e das ocupações entre os dois tipos de organização (em cadeia, independente) que acolhem clientes do segmento 'Turístico'. 
Tabela 7 - Médias Aritméticas Verificadas nos Hoteis Organizados em Cadeias e nos Hoteis Independentes, do Segmento 'Turístico'.

\begin{tabular}{lcc}
\hline Hotel Organizado em Cadeia? & $\begin{array}{c}\text { Média Aritmética das } \\
\text { Tarifas Médias Mensais }\end{array}$ & $\begin{array}{c}\text { Média Aritmética das Taxas Mensais de } \\
\text { Ocupação }\end{array}$ \\
\hline Sim & 154,00 & 65,50 \\
Não & 151,00 & 62,00 \\
\hline \hline Total & 152,50 & 63,75 \\
\hline
\end{tabular}

Notas:

Média aritmética das tarifas médias mensais: $\mathrm{O}$ montante da soma das tarifas médias mensais, dividido pelo número de meses (9).

Média aritmética das taxas mensais de ocupação: $\mathrm{O}$ montante da soma das taxas mensais de ocupação, dividido pelo número de meses (9).

Os valores da tabela são os desvios-padrão e as médias, calculadas sem considerar as não-respostas.

Resultados do teste de Fisher:

Média aritmética das tarifas mensais: entre $=9.00$, dentre $=82.00, \mathrm{~F}=0.11,1-\mathrm{p}=46.41 \%$ Média aritmética das ocupações mensais: entre $=12.25$, dentre $=66.25, \mathrm{~F}=0.19,1-\mathrm{p}=47.32 \%$ Fonte: Resultados obtidos a partir dos dados informados pelos hoteis de Belo Horizonte.

\section{Considerações Finais}

A análise dos desempenhos das tarifas e das taxas de ocupação seguiu dois nortes: o norte dos segmentos de clientes que os hoteis acolhem e o das duas formas de organização (hoteis organizados em cadeias; hoteis independentes).

Três núcleos de perguntas balizaram a pesquisa: Há diferenças nas tarifas médias aplicadas e nas ocupações obtidas pelos hoteis, dentre e entre os diversos segmentos de clientes que eles acolhem? Há diferenças nas tarifas médias aplicadas e nas ocupações obtidas dentre e entre os hoteis com distintas formas de organizar suas atividades? Em que extensão a tarifa aplicada pelo hotel determina sua taxa de ocupação?

Quanto ao desempenho das tarifas médias os resultados apontaram para a rejeição das hipóteses nulas - ausências de relações - e aceitação das hipóteses alternativas. Isto é, as tarifas apresentaram diferenças estatisticamente significativas dentre e entre os segmentos de clientes que os hoteis acolhem, bem como dentre os hoteis organizados em cadeias e entre eles e os hoteis independentes. No que concerne ao desempenho das taxas de ocupação, os 
resultados apontaram para a aceitação das hipóteses nulas e rejeições das hipóteses alternativas.

Sobre a extensão em que a tarifa aplicada pelo hotel determina sua taxa de ocupação, verificou-se: 1) Dependências estatisticamente não significativas, tanto na amostra total, como nos segmentos 'Econômico' e 'Simples'; 2) Insuficiente quantidade de relações (meses) estatisticamente significativas no segmento 'Superior'; 3) Moderados, fortes e muito fortes coeficientes de correlação - apresentando relações negativas - na maioria dos meses e nas médias aritméticas dos segmentos 'Luxo' e 'Turístico'. Contudo, tanto na amostra total, como nos segmentos 'Simples' e 'Econômico' as tarifas médias não apresentaram correlações estatisticamente significativas com as taxas de ocupação e no segmento 'Superior' houve apenas dois meses de relacionamento positivo; assim, conclui-se que há de se discriminar os segmentos e estudar as sazonalidades para se determinar a extensão em que a mudança da tarifa — aumento ou redução — afeta a taxa de ocupação do hotel.

A descoberta das correlações negativas entre as taxas de ocupação e as tarifas médias aplicadas pelos hoteis dos segmentos 'Luxo' e 'Turístico' estimulou-nos a verificar se haveria diferenças significativas dentre essas variáveis, segundo as formas que os hoteis adotam para organizar suas atividades. Os resultados apontaram para diferenças estatisticamente não significativas das tarifas e das ocupações entre os dois tipos de organização (hoteis organizados em cadeias; hoteis independentes) nos segmentos 'Luxo' e 'Turístico'.

Os resultados demonstrados neste trabalho expressam exclusivamente a realidade de Belo Horizonte, no período de dezembro de 2008 a agosto de 2009. Assim, não se recomenda aplicá-los a outras regiões e a qualquer época, isto é, a ocupação na hotelaria depende em grande parte de eventos. Entrementes, as relações negativas entre tarifas médias e taxas de ocupação apuradas com os hoteis dos segmentos 'Luxo' e 'Turístico' são semelhantes às obtidas na pesquisa conduzida por Pinto (2009) na cidade de Santos, SP.

Convém destacar, ainda, que os dados foram preenchidos pelos gestores dos hoteis pesquisados, sendo, portanto, de responsabilidade exclusiva deles, ou seja, os pesquisadores não tiveram acesso aos registros contábeis das tarifas e das ocupações. 
As descobertas apontam para a necessidade de extensão desta pesquisa para outras regiões, fundamentalmente com hoteis dos segmentos 'Luxo', 'Superior' e 'Turístico'. Com os hoteis do segmento 'Superior', para verificar a tendência da relação entre a tarifa média e a taxa de ocupação. Com hoteis dos segmentos 'Luxo' e 'Turístico', para confirmar ou negar a suposição que os resultados deste trabalho fazem emergir: "as relações entre tarifas médias e taxas de ocupação são negativas, isto é, quanto maior a tarifa aplicada, menor a taxa de ocupação". Essa necessidade se intensifica em virtude do pequeno número de hoteis classificados como 'Luxo’ e ‘Econômico’ que responderam à pesquisa.

Revelando-se com mais nitidez a relação da tarifa com a ocupação, nos segmentos indicados para pesquisa, será facilitado aos gestores desenvolverem o processo de precificação. Como alertam os pesquisadores Arbel e Woods (1991): "Na atual economia, as tradicionais estratégias e técnicas de fixação de preços usadas para determinar novas tarifas podem prejudicar drasticamente o lucro" (p. 67).

\section{Referências}

AGGETT, Mandy. What has influenced growth in the UK's boutique hotel sector? International Journal of Contemporary Hospitality Management, 19, 2: 169-177, 2007.

ARBEL, Avner; WOODS, Robert H. Inflation and hotels: The cost of following a faulty routine. The Cornell Hotel and Restaurant Administration Quarterly, 31, 4: 66-75, 1991.

BAUM, Tom; MUDAMBI. Ram. An empirical analysis of oligopolistic hotel pricing. Annals of Tourism Research, 22, 3: 501-516, 1995.

BOTELHO, Delane; URDAN, André T. Lealdade à marca e sensibilidade ao preço: um estudo da escolha da marca pelo consumidor. Revista de Administração Contemporânea, 9, 4: 163-180, 2005.

CARVELL, Steven A.; QUAN, Daniel C. Exotic reservations: low prices guarantee. International Journal of Hospitality Management, 27, 2: 162-169, 2008.

CHAN, Eric S. W.; WONG, Simon C. K. Hotel selection: when price is not the issue. Journal of Vacation Marketing, 12, 2: 142-159, 2006.

CHERNEV, Alexander. Strategic marketing management. (Fifth edition). New York: McGraw-Hill, 2009.

CHUNG, Kyoo Y. Hotel room rate pricing strategy for market share in oligopolistic competition: eight-year longitudinal study of super deluxe hotels in Seoul. Tourism Management, 21, 2: 135-145, 2000.

CRESSMAN JR., George E. Commentary on industrial pricing: theory and managerial practice. Marketing Science, 18, 3: 455-458, 1999. 
FERNANDES, M. Concepción L.; BEDIA, Ana M. S. Is the hotel classification system a good indicator of hotel quality? An application in Spain. Tourism Management, 25, 6: 771-775, 2004.

HAIR JR., Joseph F.; WOLFINBARGER, Mary; ORTINAU, David J.; BUSH, Robert P. Fundamentos de pesquisa de marketing. Tradução Francisco Araujo da Costa. Porto Alegre: Artmed, 2010.

HOOLEY, Graham; SAUNDERS, John; PIERCY, Nigel F.; NICOULAUD, Brigitte. Marketing strategy and competitive positioning. (Fourth edition). New York: Prentice Hall, 2008.

KIM, Hong-bumm; KIM, Woo G. The relationship between brand equity and firms' performance in luxury hotels and chain restaurants. Tourism Management, 26, 4: 549-560, 2005.

KOTLER, Philip. Kotler on marketing: How to create win and dominate markets. London: Simon and Schuster, 2001.

KOTLER, Philip; KELLER, Kevin L. Marketing management. (Thirteenth edition). Upper Saddle River: Pearson Prentice Hall, 2008.

LAMB, Charles W.; HAIR JR., Joseph F.; MCDANIEL, Carl. Marketing. (Seventh edition). Mason Southwestern: Irwin, 2004.

LEHMANN, Donald R.; WINER, Russell S. Product management, fourth edition. New York: Irwin, 2004.

LOCKYER, Tim. Understanding the dynamics of the hotel accommodation purchase decision. International Journal of Contemporary Hospitality Management, 17, 6: 481-492, 2005.

LOVELOCK, Christopher H.; WIRTZ, Jochen. Services marketing: people, technology, strategy. Seventh edition. Upper Saddle River: Prentice Hall, 2010.

LOVELOCK, Christopher H.; WRIGHT, Lauren. Serviços, marketing e gestão. São Paulo: Saraiva, 2005.

NAGLE, Thomas T., HOGAN, John E., ZALE, Joseph. The strategy and tactics of pricing: a guide to growing more profitability, fifth edition. Upper Saddle River: Prentice Hall, 2010.

NASUTION, Hanny N.; MAVONDO, Felix T. Customer value in the hotel industry: what managers believe they deliver and what customer experience. International Journal of Hospitality Management, 27, 2: 204-213, 2008.

PALIA, Aspy P. Target profit pricing with the web-based breakeven analysis package. Developments in Business Simulation and Experiential Learning, 35: 197-204, 2008.

PINTO, Éder P. Tarifa e ocupação em hoteis: provocando o modelo de apreçamento convencional. Revista Turismo em Análise, 20, 3: 469-484, 2009.

REST, J-P. I. Van Der; HARRIS, P. J. Optimal imperfect pricing decision-making: modifying and applying Nash's rule in a service sector context. International Journal of Hospitality Management, 27, 2: $170-178,2008$.

SCHWARTZ, Zvi. Advanced booking and revenue management: room rates and the consumers' strategic zones. International Journal of Hospitality Management, 25, 3: 447-462, 2006.

SCHWARTZ, Zvi; CHEN, ChihChien. The peculiar impact of higher room rates on customer's propensity to book. International Journal of Contemporary Hospitality Management, 22, 1: 41-55, 2010 . 
WILKINS, Hugh; MERRILEES, Bill; HERINGTON, Carmel. Towards an understanding of total service quality in hotels. International Journal of Hospitality Management, 26, 4: 840-853, 2007.

Recebido em: 02/07/2011

Aprovado em: 09/02/2012 (1 ${ }^{\text {a }}$ versão) $12 / 06 / 2012\left(2^{\mathrm{a}}\right.$ versão) 